\title{
CFD ANALYSIS OF RAE 2822 SUPERCRITICAL AIRFOIL AT TRANSONIC MACH SPEEDS
}

\author{
K.Harish Kumar ${ }^{1}$, CH.Kiran Kumar ${ }^{2}$, T.Naveen Kumar ${ }^{3}$ \\ ${ }^{1}$ M.Tech Thermal Engineering, Sanketika Institute of Technology \& Management, Vizag. \\ ${ }^{2}$ Asst. Professor, Dept of Mechanical Engineering, Sanketika Institute of Technology \& Management, Vizag. \\ ${ }^{3}$ Technical Assistant, Dept of Mechanical Engineering, Gayatri Vidya Parishad College of Engineering, Vizag.
}

\begin{abstract}
The Aerodynamic characteristics of an airfoil play a very important role in terms of design aspects and experimental validation. A distinctive supercritical airfoil is found to give inappropriate results at peak operating conditions. This led to design changes and optimization of the foil with the help of a cusp like structure added to the trailing edge of the foil. The present work emphasizes the computation of a supercritical airfoil with and without wedge profiles at different angles of attack and free stream velocities. The formation of a shock wave is observed when the free stream approaches a transonic mach number. This shock wave is a type of propagating disturbance which greatly effects the aerodynamic performance of the airfoil. The flow properties such as pressure, temperature and density experience a drastic change upstream and downstream of the generated shock. The main goal of the proposed paper is to carryout the simulation and analysis of RAE 2822 supercritical airfoil with and without wedge profiles along with the study of aerodynamic characteristics such as Lift and drag coefficients at different Mach numbers. The objective is to improve the stability of the airfoil when the flow approaches transonic Mach speeds. The design profile chosen is based upon existing literature studies.
\end{abstract}

Key Words: Supercritical Airfoil, Shock wave, Transonic mach, Mach number etc...

\section{INTRODUCTION}

There has been continuous ongoing research on the study of aerodynamic characteristics of an airfoil at different angles of incidence and different flow regimes. It is always significant to state the stability of a foil at all operating conditions. The parameters that affect the stability include Mach number, Lift \& Drag coefficients, Pressure drag and the strength of the generated shock wave. The changes in any of these parameters will result in appreciable loss in stability of the airfoil. In order to overcome the situation, many numerical simulations have been carried out for each chosen profile to bring out the best possible stability characteristics so that they can be used in many aerodynamic applications. The foils that gave poor stability characteristics have been eliminated as they account for poor lifting performance. So it is always desirable for an airfoil to possess best stability characteristics which can further achieve good lifting performance at optimum and extreme flow conditions. The present study mainly focuses on this aspect which can be further developed depending on the criteria requirements.

\subsection{Literature Review}

Research shows that the main mach regime accounting for instability of the airfoil is found to be the transonic mach regime. This is due to the generation of shockwaves at transonic mach speeds (Mach $=0.8$ to 1.2). The only solution they found for improving the stability characteristics is delaying the generation of a shock. This can be done by moving the Critical Mach number beyond the actual one by changing the design inputs. So the research moved to design a new kind of airfoil which can overcome the instability at transonic speeds. This lead to the design of a Supercritical airfoil which possesses higher thickness and more flatness of upper chamber. This new design gave good performance curves for transonic mach regime but found to have some demerits also when coming to operation at subsonic or supersonic speeds.

Novel Kumar Sahu \& Mr. Shadab Imam ${ }^{[1]}$ have carried out a simulation analysis for a transonic flow over an airfoil to give appropriate results which can further add scope of work for extended research. They have carried out the analysis inorder to reduce tragic failures due to shock wave generation. Their results show that the strength of a shock wave increases with increase in mach speed of the foil which also leads to increase in drag, both due to the emergence of wave drag, and also because the pressure rise through a shock wave thickens the boundary layer leading to increased viscous drag which limits the cruise speed. They suggested that usage of swept wings may reduce the drag upto some extent at high mach speeds and the mach induced changes in control effectiveness. They further added that increase in angle of attack results in increase of lift coefficient upto a limiting point after which an aerodynamic stall occurs. They finally concluded by stating the results that the limiting angle of attack is $16^{\circ}$ after which the foil is observed to be stalled.

P.Sethunathan, M.Niventhran, V.Siva \& R.Sadhan $\mathrm{Kumar}^{[2]}$ have carried out a analysis on different supercritical airfoils like NACA 0406, 0412, 0706 and 1006 to show the improvement in the climbing performance of the foils at 
subsonic mach speeds. He showed that a cusp like structure at the trailing edge of an unsymmetrical airfoil produces a very high improvement in climbing performance. The test is carried out at a subsonic speed of around $25 \mathrm{~m} / \mathrm{s}$ and the results so obtained show the reduction in drag and improvement in coefficient of lift by $15-20 \%$ when compared with baseline model.

A.B.M Toufique Hasan \& Md. Mahbub Alam ${ }^{[3]}$ have used Reynolds Averaged Navier-Stokes (RANS) equations to predict the transonic buffet and corresponding aerodynamic behavior over NASA SC(2) 0714 supercritical airfoil. RANS computations have been performed at a free stream Mach no of 0.77 while varying the angle of attack from $2^{\circ}$ to $7^{\circ}$. The computation results so obtained have been validated with experimental results. The results showed that no shock oscillation is observed till $2^{\circ}$ angle of attack and resulted in a transonic buffet from $3^{\circ}$ to $7^{\circ}$ for the same Mach speed. It was shown that shock oscillating zone and the intensity of shock-boundary layer interaction is increased with rise in angle of attack. Finally the study was concluded by saying that unsteady shock movement creates fluctuations in lift, drag and static pressure over the upper surface of the airfoil.

Mochammad Agoes Moelyadi ${ }^{[4]}$ has done the trailing edge modifications by employing different wedge profiles. He has carried out simulation for RAE 2822 transonic airfoil by changing the wedge shapes at the aft portion of the foil. He has taken two different wedge configurations where he has changed the length to height ratios and concluded by saying that the airfoil with wedge having a length of $1 \%$ of the chord and height equal to $0.5 \%$ is giving good aerodynamic performance in comparison with foil with other wedge profiles. The six different wedge profiles under two configurations ratio wise for which the experiments were conducted are shown in fig 1.

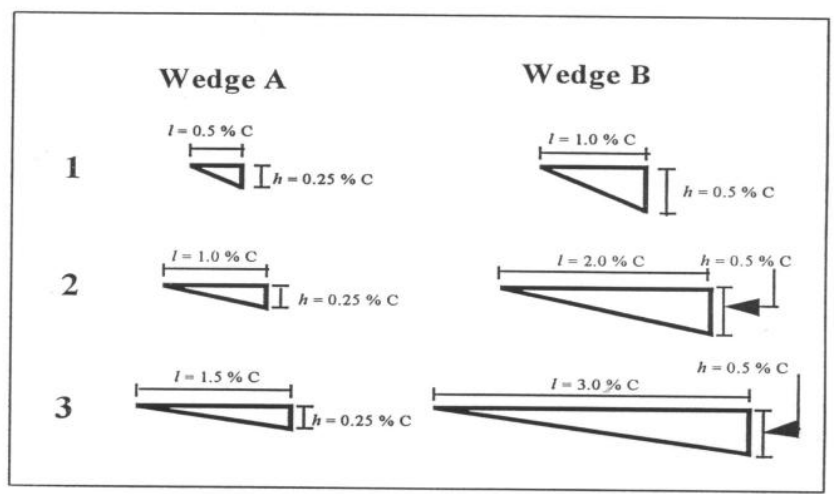

Fig-1: Six Wedge Configurations used to improve aerodynamic performance of Supercritical airfoil

\subsection{Objective of the Work}

The main objective of the thesis focuses on the development and study of existing airfoil theories that are applicable at transonic and supersonic mach regimes. The development led to changes in the design of the foil thus extending the boundaries of operating angles of attack and resulted in achieving good aerodynamic stability characteristics. The other objectives of the thesis include:
- Visualizing the flow over a airfoil at different mach regimes

- Study of existing methods of simulation

- Optimizing the design requirements

- Explanation of design and construction features

- Understanding the changes in flow parameters and boundary conditions

- $\quad$ Plotting of results accordingly

- Comparison of theoretical and simulation results

- Discussion of thesis development and future scope

\section{MODELING}

The modeling and design of RAE 2822 airfoil is done using CATIA v5 R20. The CFD Solver chosen to carryout the Simulation work is ANSYS FLUENT. CFD Analysis is widely preferred to carryout the simulation works involving fluid flows. This makes use of algorithms and numerical techniques to solve various aerodynamic, marine and fluid flow problems.

The designing of RAE 2822 supercritical airfoil is done by importing the airfoil coordinates from an airfoil tool generator. The coordinates are imported to CATIA v5 R20 by running a macro file and the transonic airfoil is generated by joining all the coordinates.

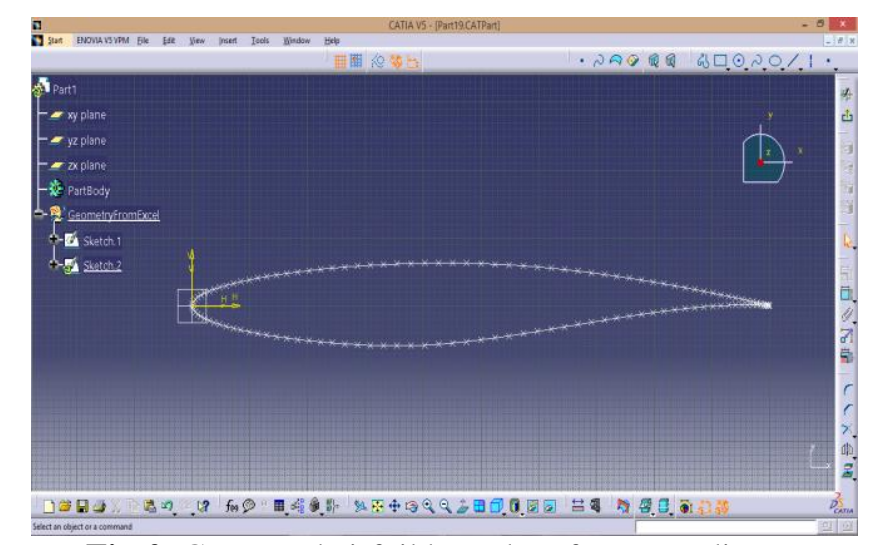

Fig-2: Generated airfoil boundary from coordinates

After running the macro file, the generated $3 \mathrm{D}$ points are projected onto a $2 \mathrm{D}$ plane and the generated $2 \mathrm{D}$ points are joined using a spline thus forming the upper and lower cambers of the airfoil which completes the boundary.

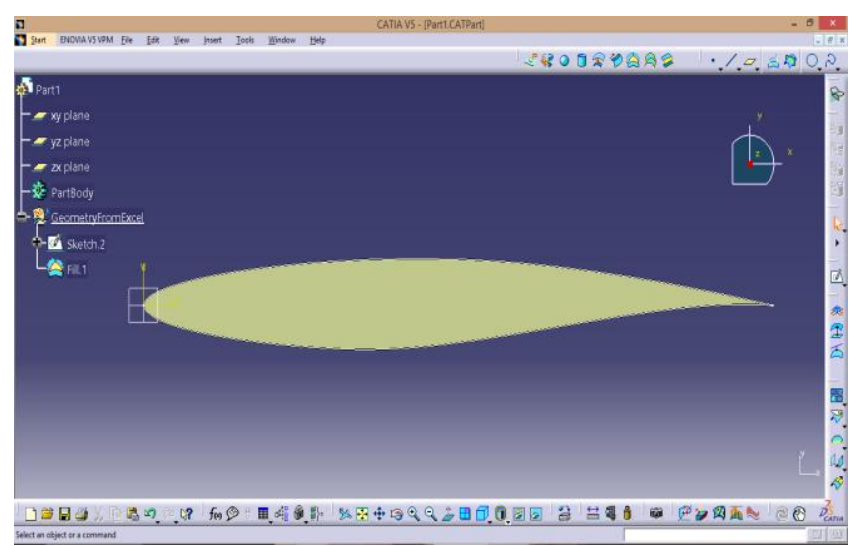

Fig-3: Generated Airfoil Surface from the Coordinates 


\section{ANALYSIS}

ANSYS Workbench 15.0 is used for the analysis and the solver chosen is Fluent. The generated Catia model is imported into Ansys and the domain and region of influence are constructed in the geometry part of the Fluent module.

The inner and outer domains are shown in Fig 4. The inner domain was fine meshed and outer was coarse meshed. The inputs given for meshing are stated below.

Table -1: Details and Sizing inputs of a mesh

\begin{tabular}{|l|l|}
\hline Physics Preference & CFD \\
\hline Solver Preference & Fluent \\
\hline Advanced size function & On: Proximity \& Curvature \\
\hline Relevance center & Fine \\
\hline Smoothing & High \\
\hline Maximum face size & $0.1 \mathrm{~m}$ \\
\hline Maximum size & $0.1 \mathrm{~m}$ \\
\hline Growth rate & 1.2 \\
\hline
\end{tabular}

In the sizing option set the element size as $0.3 \mathrm{~m}$ and growth rate as 1.2. The sizing and inflation inputs are as follows

Table -2: Details and Inflation inputs of a mesh

\begin{tabular}{|l|l|}
\hline Inflation Option & Total thickness \\
\hline No of layers & 5 \\
\hline Growth rate & 1.2 \\
\hline Maximum thickness & $0.02 \mathrm{~m}$ \\
\hline
\end{tabular}

Create named selection for all the surfaces as inlet1, outlet1, uppercamber, lowercamber, symmetry1 and symmetry2. Wait until the meshing is done and a pop up appears saying meshing done.

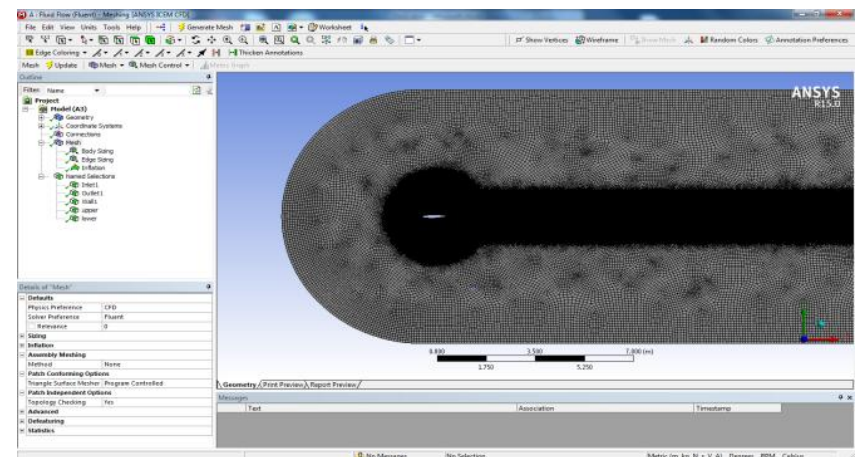

Fig-4: Meshed areas showing inner and outer domains along with region of influence.

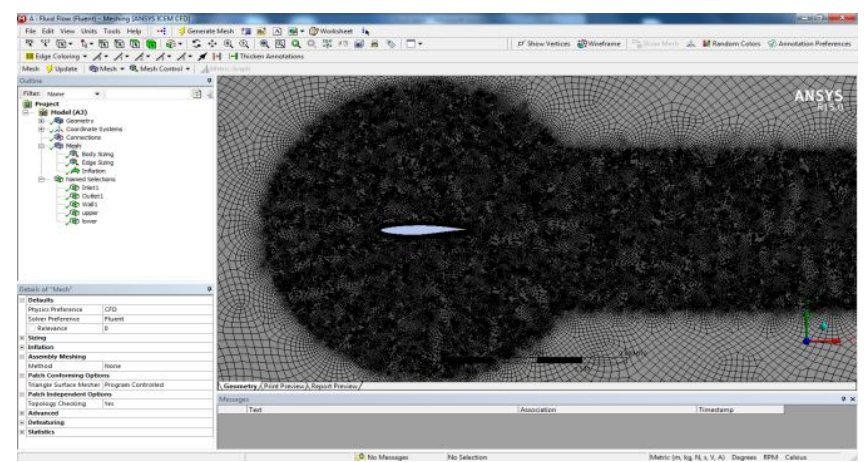

Fig-5: Meshed areas showing coarse and fine mesh domains
As the flow simulation involves higher accuracy, fine mesh and least element sizes are used around the foil keeping all other input values as default. Also the analysis requires higher number of iterations to converge as the simulation work involves operation of the airfoil at wide ranges of speeds ranging from subsonic to supersonic. The visualization of shocks and flow contours is also important and hence the iterations done for each analysis are chosen to be 3000 .

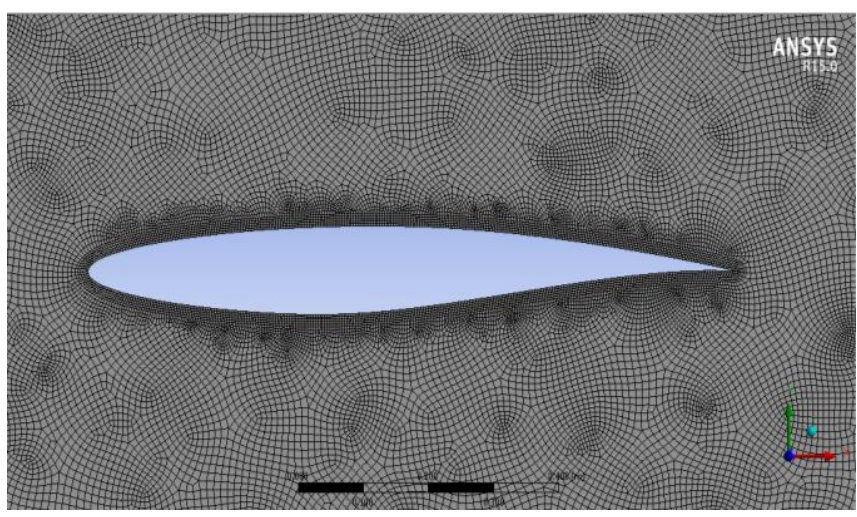

Fig-6: Meshed area showing fine mesh around airfoil

Next is the Solution Setup where the solver inputs must be given and the type chosen here is density based and steady state analysis. The turbulence model used is $\mathrm{k}-\mathcal{E}$ model and standard wall functions is used as a input for Near-wall treatment. Choose air as the working medium keeping its properties unchanged.

In the boundary conditions tab, give the inlet condition as pressure-far-field and provide the necessary inputs such as Guage pressure, Mach number and select the type for given named selections. The upper camber and lower camber types are selected as wall and outlet type as pressure outlet. Select type as interior for interior-surface body and symmetry for outer domain walls.

In the reference values tab select compute from inlet and initialize the solution by selecting the reference zone as surface_body. Now go to monitors tab, select lower and upper wall zones and create lift and drag monitors. Next go to Solution initialization tab, select Standard initialization mode and initialize the solution by selecting compute from inlet. At the last, go to Run Calculation tab, give the number of iterations as 3000 and wait until the solution gets converged. Note the residual values and go for postprocessing.

The Post-processing stage comprises of visualizing the pressure, velocity, temperature and turbulence kinetic energy contours and respective plots along with scaled residuals. To check the contours in the result section go to user location and plots and click on insert contour and select the variable and number of contours that have to be displayed. Higher the number of contours give more accurate indication of variations in pressure, velocity, temperature and turbulence kinetic energy. 


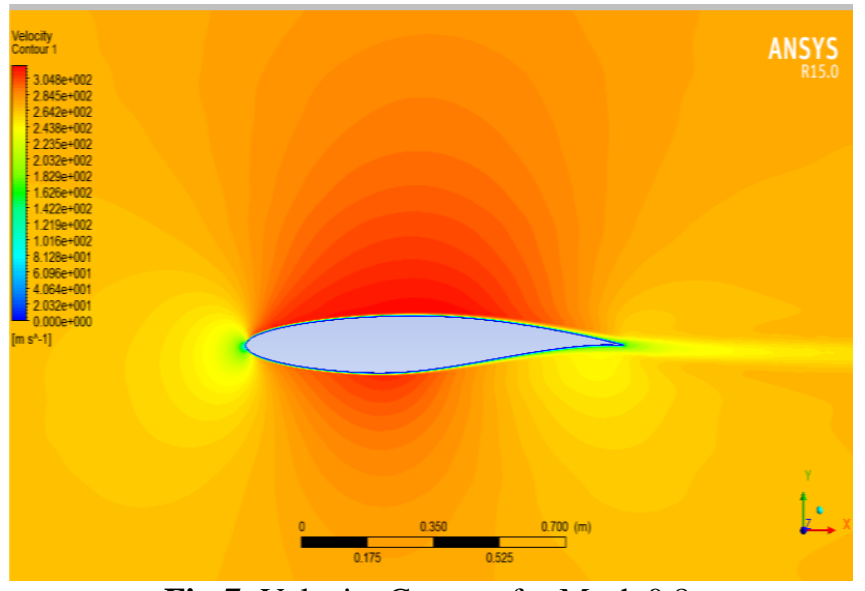

Fig-7: Velocity Contour for Mach 0.8

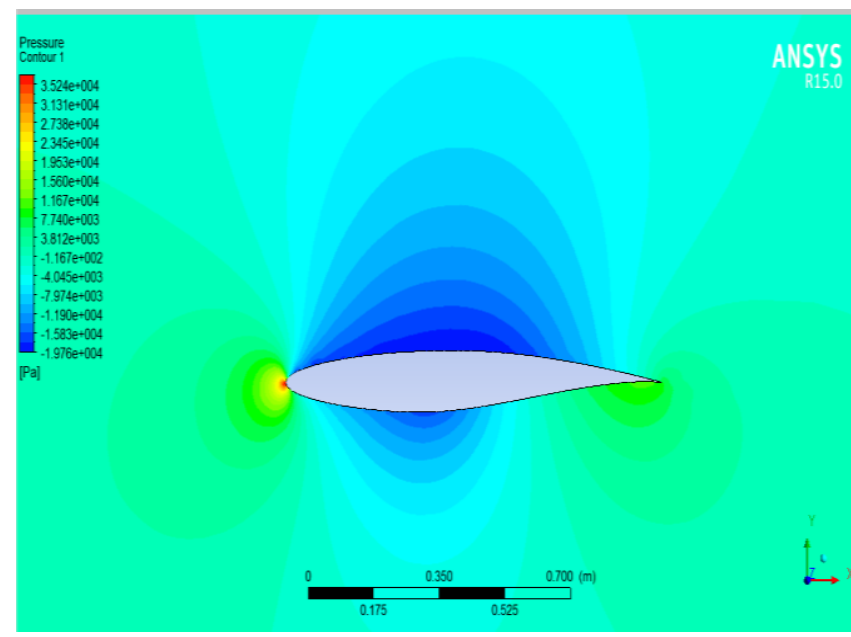

Fig-8: Pressure Contour for Mach 0.8

The Velocity and Pressure Contours show that the free stream velocity over the upper camber of the airfoil is higher when compared with that of velocity on the lower side. According to Bernoulli's principle the pressure will be higher on the bottom side of the foil and lower on the top side which are validated by the analytic contours shown in the figures 6 and 7. This is in accordance with airfoil theory where Bernoulli's principle plays a major role in lifting performance of the airfoil.

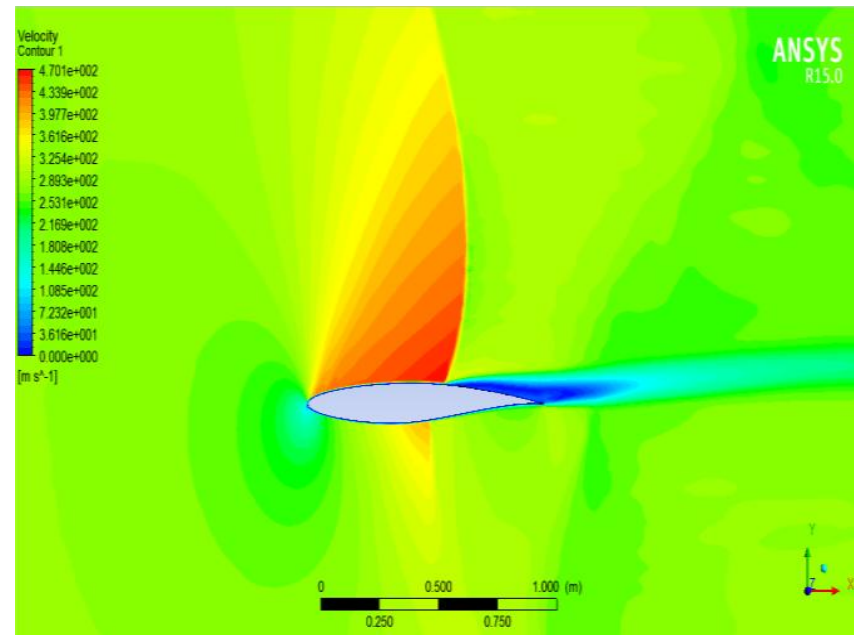

Fig-9:Velocity Variation across Normal Shock $(\mathrm{M}=0.8)$

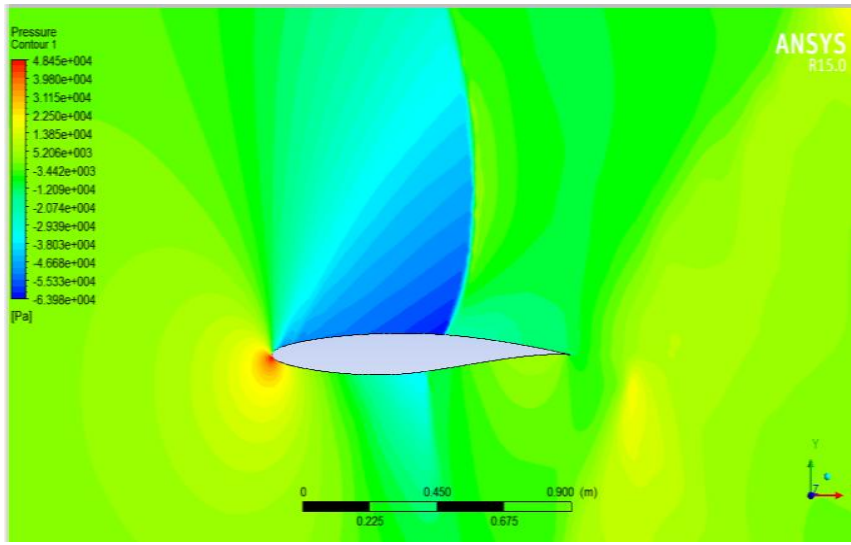

Fig-10: Pressure Variation across Normal Shock $(\mathrm{M}=0.8)$

When the speed of the aircraft approaches transonic mach regime, there will be generation of normal shock over the upper and lower cambers of the airfoil. These contours have been shown in figures 8 and 9 . The flow parameters such as pressure, velocity, temperature and density change drastically across the generated shock. With the increase of mach speed further, the strength of the generated shock increases resulting in the formation of bow shock or oblique shock when the speed approaches mach 1 . The formation of these shocks and increase in turbulence over the upper camber at the aft portion are shown in the following contour plots.

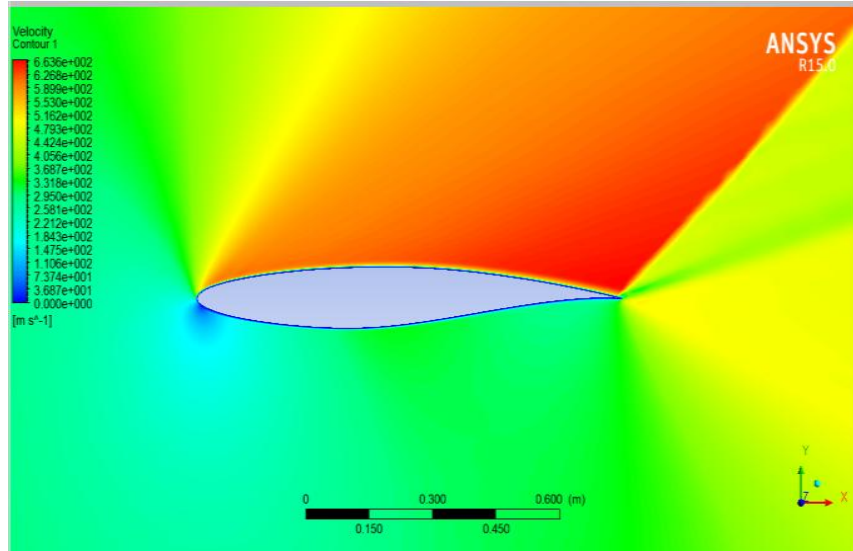

Fig-11: Velocity Variation across Oblique Shock $(\mathrm{M}=1.2)$

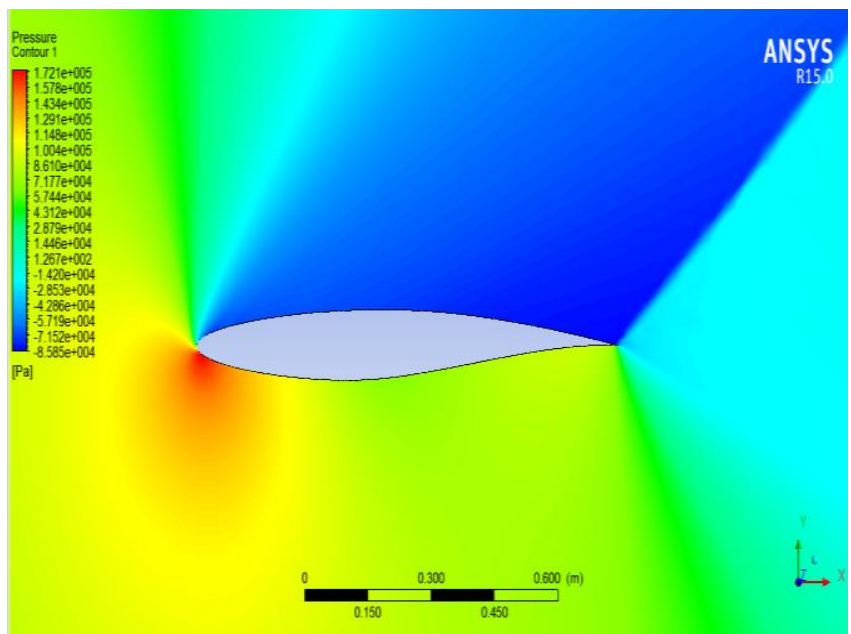

Fig-12: Pressure Variation across Oblique Shock $(\mathrm{M}=1.2)$ 


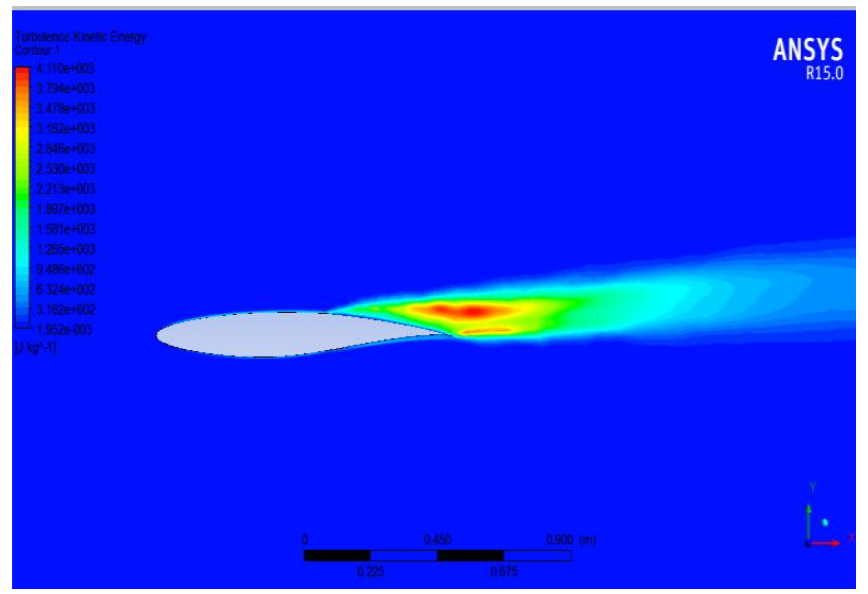

Fig-13: Turbulence Kinetic Energy Contours

\section{RESULTS AND DISCUSSIONS}

The graphs below (Charts $1 \& 2$ ) show the variation of Lift Coefficient with Mach number for different angles of attack of $5^{\circ}, 10^{\circ}$ and $15^{\circ}$. The plots show that the lift is dropping in transonic mach region from mach 0.7 to mach 1.4 . The lift produced for higher angles of attack is more when compared with those lift coefficients generated at $5^{\circ}$ and $10^{\circ}$ angles of attack. The trend followed by curves are in accordance with airfoil theory.

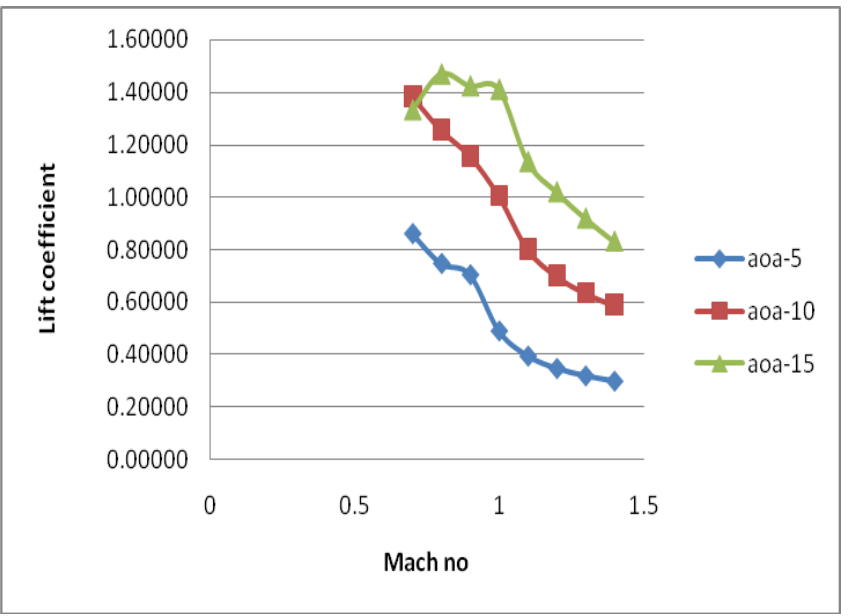

Chart-1: Lift Coefficient vs. Mach (Without Wedge)

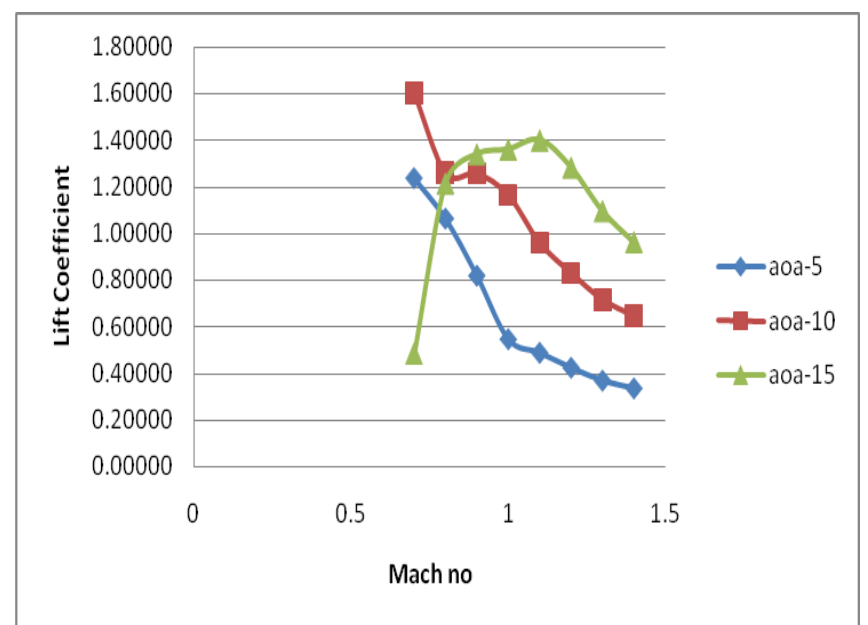

Chart-2: Lift Coefficient vs. Mach (With Wedge)
A comparison is made between the lift coefficients obtained with and without wedge profiles for a constant angle of attack. The trends in charts 3,4 \& 5 show that the Lift produced for airfoil with wedge profile is higher than that of foil without wedge.

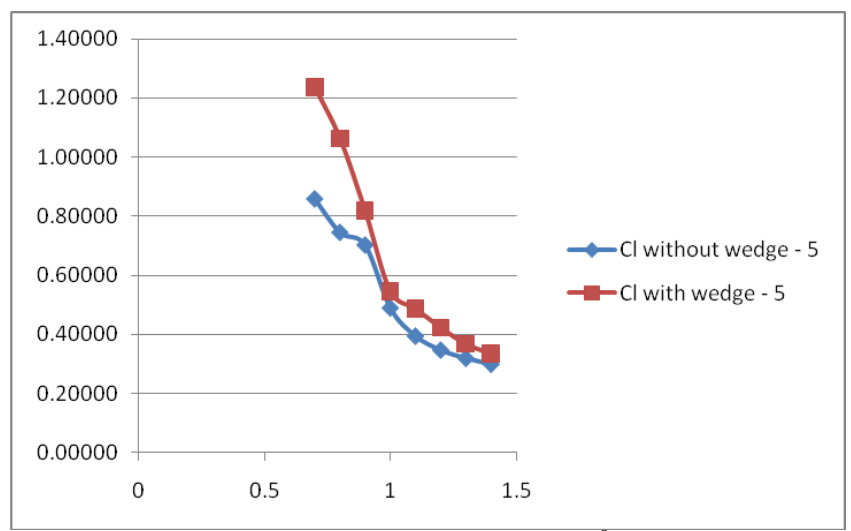

Chart-3: Lift Coefficient vs. Mach for $5^{0}$ angle of attack (With and Without Wedge Comparison)

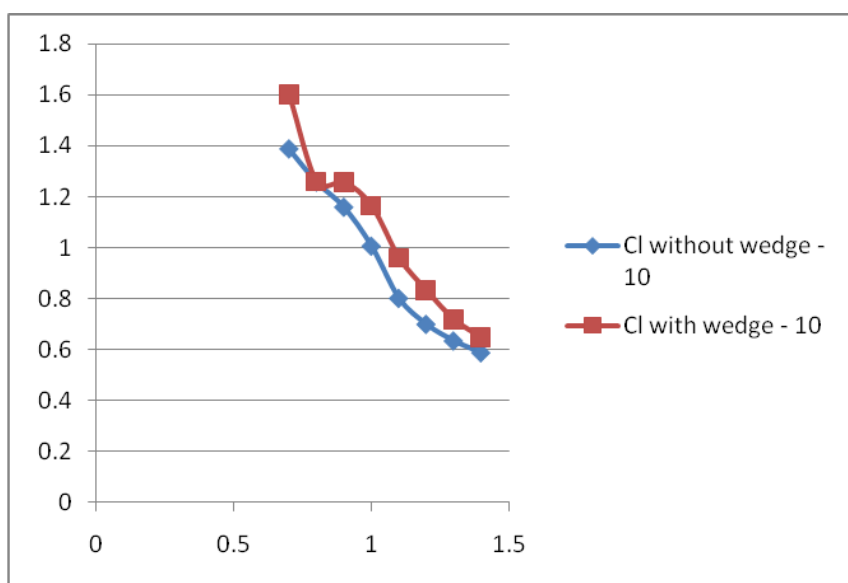

Chart-4: Lift Coefficient vs. Mach for $10^{0}$ angle of attack (With and Without Wedge Comparison)

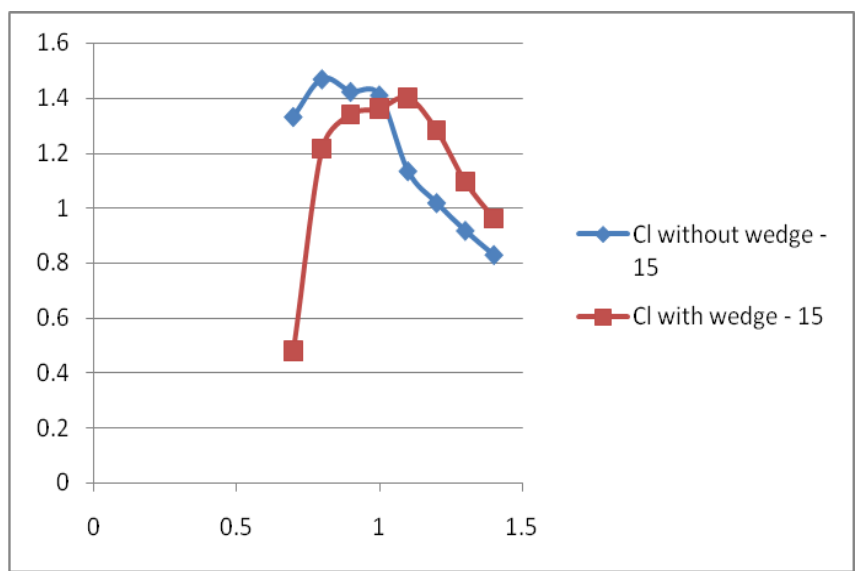

Chart-5: Lift Coefficient vs. Mach for $15^{0}$ angle of attack (With and Without Wedge Comparison)

The trend at $15^{\circ}$ angle of attack shows that the drop in lift coefficient in case of airfoil with wedge is occurring at a Mach of 1.1 when compared with foil without wedge which is occurring sooner at a mach of 0.8 . This clearly shows that the foil with wedge profile is more stable at $15^{\circ}$ angle of 
attack than the foil without wedge profile. This is due to the fact that the shock generation is delayed in the foil with wedge profile at $15^{\circ}$ angle of attack. The only drawback here is the maximum lift produced is less in case of foil with wedge but is more stable at stalling conditions.

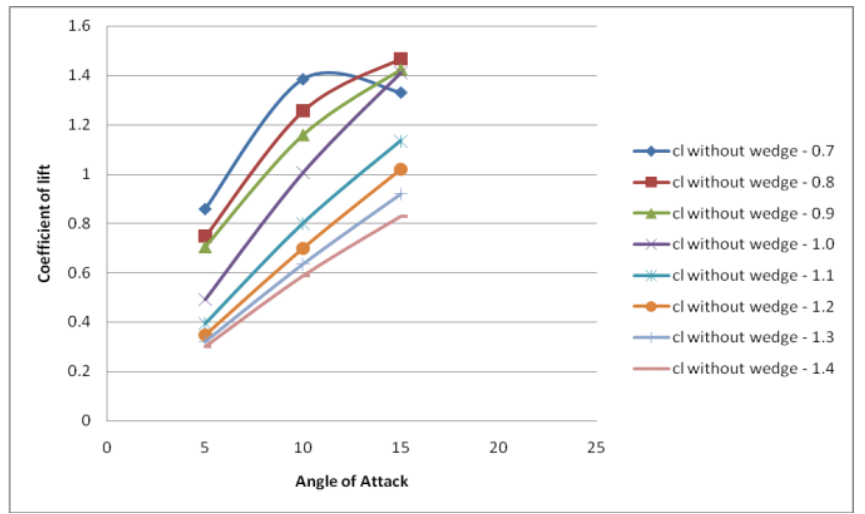

Chart-6: Lift Coefficient vs. Angle of attack

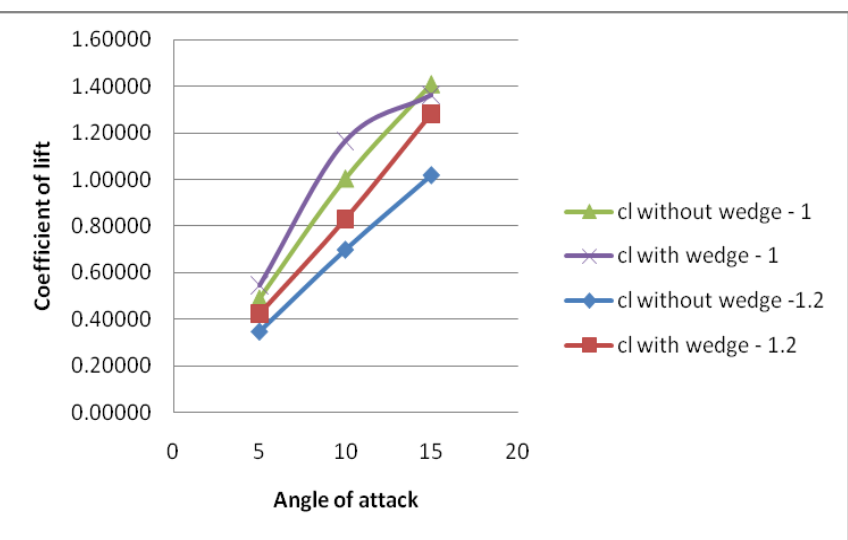

Chart-7: Lift Coefficient vs. Angle of Attack for Mach 1 \& 1.2 (With and Without Wedge Comparison)

Chart-6 shows that Lift coefficient is increasing with increase of angle of attack and is lesser for higher mach speeds. This is due to increase in drag coefficient with increase in flow speed. A comparison plot is shown in chart-7 where the trend shows that airfoil with wedge profile gives better lift performance.

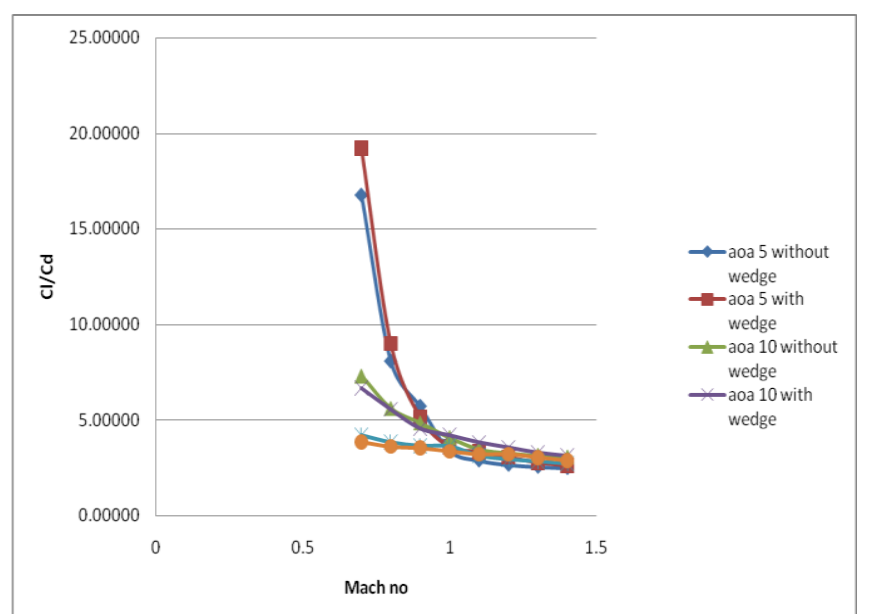

Chart-8: $\mathrm{C}_{\mathrm{l}} / \mathrm{C}_{\mathrm{d}}$ vs. Mach number (with and without wedge)
Along with increase in lift there will be proportional increase in drag also for airfoil operating at different flow regimes. For this reason, the aerodynamic performance deciding factor is considered to be the ratio of lift coefficient to drag coefficient $\left(\mathrm{C}_{\mathrm{l}} / \mathrm{C}_{\mathrm{d}}\right)$. Chart- 8 shows the change of $\mathrm{C}_{1} / \mathrm{C}_{\mathrm{d}}$ ratio with increase in mach speed. The plots for with and without wedge profiles are compared which finally showed that both the curves followed a similar trend thus stating that the performance of both the airfoils is same at transonic mach regime. There is no considerable raise in performance but an increase in stability is found in foil with wedge profile. As the criteria is mainly about the stability at transonic regimes, so the foil with wedge is said to be more stable though the overall performance is equal.

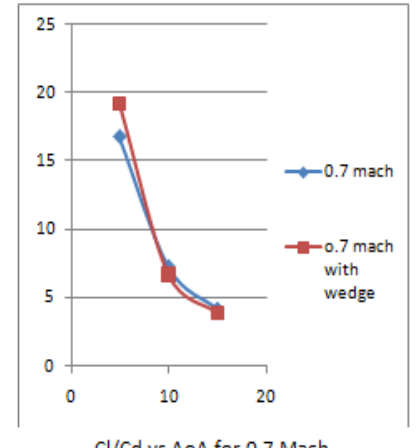

$\mathrm{Cl} / \mathrm{Cd}$ ss AoA for 0.7 Mach

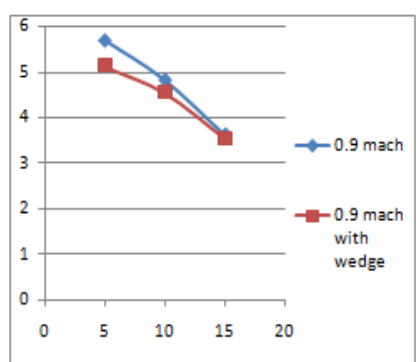

Cl/Cd vs. AQA for 0.9 Mach

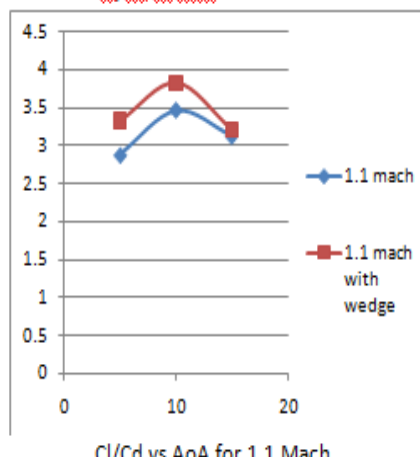

Cl/Cd us. AoA for 1.1 Mach

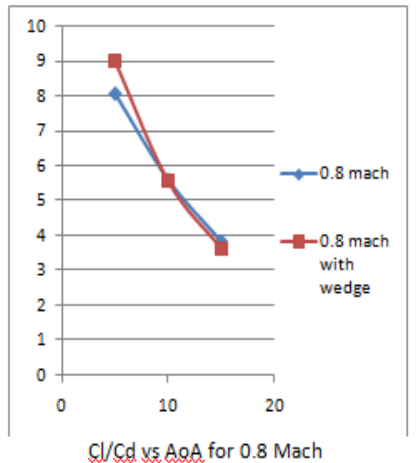

$\mathrm{Cl} / \mathrm{Cd}$ vs $\mathrm{AoA}$ for $0.8 \mathrm{Mach}$

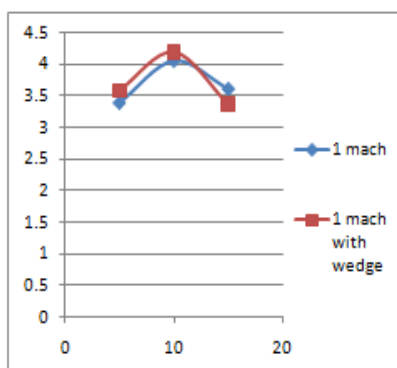

$\mathrm{Cl} / \mathrm{Cd}$ ys AQA for $1.0 \mathrm{Mach}$

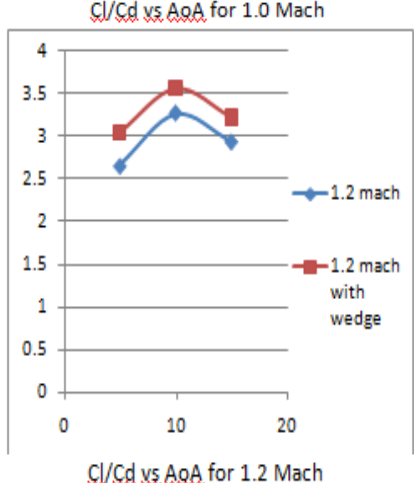

Cl/Cd ys AoA for 1.2 Mach
Chart-9: $C_{1} / C_{d}$ vs. Angle of Attack

Chart-9 shows the variations of $C_{1} / C_{d}$ with respect to angle of attack for each mach number. A comparison is made between the performance of airfoils with and without wedge profiles. The trends show that performance is higher for foil with wedge upto $10^{\circ}$ angle of attack thereafter which the performance drops. The same trend is continued upto mach speed equal to 1 . After crossing mach 1 it is observed that the foil with wedge profile is giving higher performance at all angles of attack. This clearly shows that the overall performance can be increased when the airfoil is operated at 
an angle less than $10^{\circ}$ till sonic mach is reached and upto stall angle after crossing sonic mach.

\section{CONCLUSIONS}

$>$ The Lift Coefficient is increasing with increase of angle of attack and decreasing for increase in mach speed due to rise in drag coefficient in the transonic region. This is in agreement with airfoil theory.

$>$ The Maximum Lift obtained in the Transonic airfoil with wedge profile is relatively less but is more stable at 15 degrees angle of attack which is near to the stall point.

> The Pressure, Velocity, Temperature \& Turbulence Kinetic Energy Contours are found to be appropriate for corresponding Mach inputs and angles of attack.

$>$ The ratio of Lift to Drag coefficient determines the airfoil performance which follows the same trend for both the foils.

$>$ The Plots with respect to angle of attack show that the foil with wedge performs better upto an angle of attack of 10 degrees and drops on further increase in angle of attack. This trend is continued upto Mach 1.

$>$ The trend after Mach 1 shows that the foil with wedge gives better performance at all angles of attack.

"The airfoil with wedge profile is more stable and contributes for better performance for operation at an angle less than 10 degrees upto Mach 1 and upto stall angle beyond Mach 1. This Shows that Supercritical airfoil with wedge profile is more stable and gives good performance characteristics at transonic mach regime"

\section{REFERENCES}

[1]. Novel Kumar Sahu, Mr. Shadab Imam., "A Review on Transonic Flow over an Airfoil", International Journal of Innovative Science, Engineering \& Technology, Vol. 2, Issue 5, May 2015.

[2]. P.Sethunathan, M.Niventhran, V.Siva \& R.Sadhan Kumar., "Analysis of Aerodynamic Characteristics of a Supercritical Airfoil for Low Speed Aircraft", International Journal of Research in Engineering and Technology, eISSN: 2319-1163, pISSN: 2321-7308.

[3]. A.B.M Toufique Hasan \& Md. Mahbub Alam., "RANS computation of transonic buffet over a supercritical airfoil", $5^{\text {th }}$ BSME International Conference of Thermal Engg, published by Elsevier Ltd., 2013.

[4]. Mochammad Agoes Moelyadi., "Improvement of transonic aerofoil Aerodynamic performance with trailing Edge modification using wedge Configuration", ICAS 2002 Congress.

[5]. Hoang Thi Bich Ngoc \& Nguyen manh Hung., "Study of Separation Phenomenon in Transonic Flows Produced by Interaction between Shock Wave and Boundary layer" , Vietnam Journal of Mechanics, VAST, Vol. 33, No.3 (2011), pp. 170-181.

[6]. J.E.Michael \& M.A.R.Shariff., "Effect of Surface Roughness on Turbulent Transonic Flow around RAE2822 Airfoil", International conference on Mechanical Engineering 2005 (ICME 2005), Dec 2005.
[7]. P.R.Ravi Shankar, H.K.Amarnath \& Omprakash D Hebbal., "Simulations of Supercritical Airfoil at different Angle's of Attack with a simple Airfoil using Fluent", International journal of Engineering Reasearch \& Technology(IJERT), ISSN: 2278-0181, Vol. 3, Issue 8, Aug 2014.

[8]. Alexander Kuzmin., "Sensitivity Analysis of Transonic Flow over J-78 Wings", International Journal of aerospace Engineering, Volume 2015, Article Id 579343, Dec 2014.

[9]. Abhay Sharma, Manpreet Singh Sarwara, Harsimranjeet Singh, Lakshya Swarup, Rajeev Kamal Sharma., "CFD and Real Time Analysis of an Unsymmetrical Airfoil”, IJRMET Vol.4, Issue 2, MayOct 2014, ISSN: 2249-5762.

[10]. Mellisa B.Rivers \& Richard A.Wahls., "Comparison of Computational and Experimental results of a Supercritical Airfoil”, NASA Technical Memorandum 4601, Langley Research Center, Virginia, Nov 1994. 